\title{
COMMON FIXED POINTS AND PARTIAL ORDERS
}

\author{
ARNE BRØNDSTED
}

ABSTRACT. It is observed that certain theorems on common fixed points may be derived from a theorem on partially ordered sets.

In a previous note [2] we explained how certain fixed point theorems are direct consequences of certain theorems on partially ordered sets. In fact, let $(E,<)$ be a partially ordered set which admits at least one maximal element $x_{0}$, and let $f$ be a self-mapping of $E$ such that $x \leqslant f(x)$ for all $x \in E$; then $f\left(x_{0}\right)=x_{0}$. In the present note (which is entirely conceptual) we shall develop this point of view a little further; our motivation has mainly been the appearance of [6].

Notation. Everywhere in the following $(E, d)$ is a complete metric space, < is a partial order on $E$ (i.e., $\leqslant$ is reflexive, transitive and asymmetric), and $\varphi$ is a real valued function on $E$ which is bounded below. For $x \in E$ we denote by $S(x, \leqslant)$ the set of points $y \in E$ such that $x<y$.

We shall base our considerations on the following variant of Theorem 1 of [1]:

Proposition. Assume that

(a) $\varphi$ is decreasing with respect to $\leqslant$, i.e., $x \leqslant y$ implies $\varphi(x) \geqslant \varphi(y)$;

(b) for all $\epsilon>0$ there exists $\delta>0$ such that $x<y$ and $\varphi(x)-\varphi(y)<\delta$ implies $d(x, y)<\epsilon$.

Then there exists a sequence $\left(x_{n}\right)_{n \in N}$ in $E$ (where $x_{1}$ may be taken arbitrary) and a point $x_{0} \in E$ such that

(c) $x_{n}<x_{n+1}$ for all $n \in \mathrm{N}$, and $x_{n} \rightarrow x_{0}$;

(d) $y_{n} \rightarrow x_{0}$ for all sequences $\left(y_{n}\right)_{n \in \mathrm{N}}$ with $x_{n} \leqslant y_{n}$.

Furthermore,

(e) if $x_{n} \leqslant x_{0}$ for all $n \in \mathbf{N}$, then $x_{0}$ is maximal in $(E, \leqslant)$.

Proof. We define $\left(x_{n}\right)_{n \in N}$ inductively. Take $x_{1} \in E$ arbitrary. When $x_{1}, x_{2}, \ldots, x_{n}$ have been chosen, let $a_{n}:=\inf \varphi\left(S\left(x_{n}, \leqslant\right)\right)$, and take $x_{n+1} \in$ $S\left(x_{n},<\right)$ such that $\varphi\left(x_{n+1}\right) \leqslant a_{n}+n^{-1}$. Then $x_{n} \leqslant x_{n+1}$, and for any $y \in$ $S\left(x_{n},<\right)$ we have

$$
a_{n-1} \leqslant a_{n} \leqslant \varphi(y) \leqslant \varphi\left(x_{n}\right) \leqslant a_{n-1}+(n-1)^{-1} .
$$

In particular, $0 \leqslant \varphi\left(x_{n}\right)-\varphi\left(x_{m}\right) \leqslant(n-1)^{-1}$ for $n \leqslant m$. Using (b) we then

Received by the editors April 26, 1978 and, in revised form, January 9, 1979.

AMS (MOS) subject classifications (1970). Primary 47H10. 
see that $\left(x_{n}\right)_{n \in N}$ is a Cauchy sequence, which by completeness converges, $x_{n} \rightarrow x_{0}$. Hence, (c) is proved. Using (b) it also follows from (*) that the diameter of $S\left(x_{n},<\right)$ tends to 0 as $n$ tends to $\infty$. Therefore, we also have $y_{n} \rightarrow x_{0}$ when $y_{n} \in S\left(x_{n},<\right)$, whence (d) is proved. Finally, suppose that $x_{n}<x_{0}$ for all $n \in \mathbf{N}$, and let $y \in E$ be such that $x_{0}<y$. Then we also have $x_{n}<y$ for all $n \in \mathbf{N}$ by (c). Taking $y_{n}:=y$ for all $n \in \mathbf{N}$, (d) then shows that $y=x_{0}$, i.e., $x_{0}$ is maximal. So (e) is proved.

Remark 1. The proof of the Proposition is based on a standard argument, cf. Remark 1 of [1] and the proof of Lemma 1.1 of [5]. The argument also appears in [6] and [7].

Notation. Define a partial order $<_{d, \varphi}$ on $E$ by letting $x<_{d, \varphi} y$ if and only if $d(x, y)<\varphi(x)-\varphi(y)$, cf. [1], [2]. Call a self-mapping $f$ of $E$ admissible if $x<_{d, \varphi} f(x)$ for all $x \in E$, and call a family $F$ of self-mappings of $E$ admissible if each $f \in F$ is admissible. For a set $F$ of self-mappings of $E$ define a relation $<_{F}$ on $E$ by letting $x \leqslant_{F} y$ if and only if $x=y$ or $y=$ $f_{n} \circ \ldots \circ f_{1}(x)$ for suitable $f_{1}, \ldots, f_{n} \in F$. (Clearly, $<_{F}$ is reflexive and transitive. If $F$ is admissible, then $<_{F}$ is finer than $<_{d, \varphi}$, i.e., $x<_{F} y$ implies $x<_{d, \varphi} y$, and therefore in this case $\leqslant_{F}$ is also asymmetric, and hence a partial order.) For a set $F$ of self-mappings of $E$ let $F^{*}$ denote the set of finite compositions $f_{n} \circ \ldots \circ f_{1}$ of mappings $f_{1}, \ldots, f_{n} \in F$. (Note that $x<_{F} y$ if and only if $x<F_{*} y$. Also note that if $F$ is closed under finite compositions, i.e., $F=F^{*}$, then $x \leqslant F y$ if and only if $x=y$ or $y=f(x)$ for some $f \in F$.) Call $F^{*}$ closed under countable compositions if for each sequence $\left(f_{n}^{*}\right)_{n \in N}$ of mappings $f_{n}^{*} \in F^{*}$ and each $x \in E$ such that $f_{n}^{*}(x) \rightarrow y$ for some $y \in E$, there exists $g^{*} \in F^{*}$ such that $g^{*}(x)=y$.

TheOREM. Let $F$ be an admissible set of self-mappings of E. Assume that at least one of the following conditions holds:

(1) $\varphi$ is lower semicontinuous.

(2) $F^{*}$ is closed under countable compositions.

(3) Each $f \in F$ is continuous.

Then there is a common fixed point $x_{0}$ for all mappings $f \in F$.

Proof. In case (1) we shall apply the Proposition to the partial order $<_{d, \varphi}$. We first note that (a) and (b) hold for $\leqslant_{d, \varphi}$ by the definition of $<_{d, \varphi}$. Therefore, the Proposition is applicable, let $\left(x_{n}\right)_{n \in \mathrm{N}}$ and $x_{0}$ be as described in (c)-(e). Now, note that the sets $S\left(x, \leqslant_{d, \varphi}\right)$ are closed by (1). Therefore, since $x_{m} \in S\left(x_{n}, \leqslant_{d, \varphi}\right)$ for $m \geqslant n$, it follows that $x_{0} \in S\left(x_{n}, \leqslant_{d, \varphi}\right)$, i.e., $x_{n} \leqslant_{d, \varphi} x_{0}$ for all $n \in \mathbf{N}$. By (e) it next follows that $x_{0}$ is maximal in $\left(E,<_{d, \varphi}\right)$. Then $x_{0}$ is also maximal in $\left(E, \leqslant_{F}\right)$, since $\leqslant_{F}$ is finer than $\leqslant_{d, \varphi}$. This shows that $f\left(x_{0}\right)=x_{0}$ for all $f \in F$.

In cases (2) and (3) we shall apply the Proposition to the partial order $<_{F}$. Since (a) and (b) hold for $\leqslant_{d, \varphi}$, and $\leqslant_{F}$ is finer than $\leqslant_{d, \varphi}$, (a) and (b) also hold for $<_{F}$. So, the Proposition is applicable, let $\left(x_{n}\right)_{n \in N}$ and $x_{0}$ be as described in (c)-(e). 
In case (2), suppose that there exists a subsequence $\left(x_{n}^{\prime}\right)_{n \in N}$ of $\left(x_{n}\right)_{n \in N}$ such that $x_{n}^{\prime} \neq x_{n+1}^{\prime}$ for all $n \in \mathbf{N}$. Then for each $n$ there exists $f_{n}^{*} \in F^{*}$ such that $x_{n+1}^{\prime}=f_{n}^{*}\left(x_{n}^{\prime}\right)$, whence

$$
x_{n+p}^{\prime}=f_{n+p-1}^{*} \circ \cdots \circ f_{n}^{*}\left(x_{n}^{\prime}\right)
$$

for all $n, p \in \mathbf{N}$. Since $x_{n+p}^{\prime} \rightarrow x_{0}$ for $p \rightarrow \infty$, it follows from (2) that there exists $g_{n}^{*} \in F^{*}$ such that $g_{n}^{*}\left(x_{n}^{\prime}\right)=x_{0}$, whence $x_{n}^{\prime}<_{F} x_{0}$. But then clearly we also have $x_{n}<_{F} x_{0}$ for all $n \in \mathbf{N}$. On the other hand, if no such subsequence $\left(x_{n}^{\prime}\right)_{n \in \mathbf{N}}$ exists, then $x_{m+p}=x_{m}$ for some $m \in \mathbf{N}$ and all $p \in \mathbf{N}$. Since $x_{m+p} \rightarrow$ $x_{0}$ for $p \rightarrow \infty$, we see that $x_{m+p}=x_{0}$ for some $m$ and all $p$. But then also in this case we have $x_{n}<_{F} x_{0}$ for all $n \in \mathrm{N}$. By (e) we then see that $x_{0}$ is maximal in $\left(E, \leqslant_{F}\right)$, and therefore $f\left(x_{0}\right)=x_{0}$ for all $f \in F$.

In case (3), let $f \in F$, and take $y_{n}:=f\left(x_{n}\right)$. By (d) we then have $f\left(x_{n}\right) \rightarrow x_{0}$. But we also have $f\left(x_{n}\right) \rightarrow f\left(x_{0}\right)$ by (c) and the assumption (3). Therefore, $f\left(x_{0}\right)=x_{0}$ for all $f \in F$.

REMARK 2. Case (1) is only a slight extension of the so-called Caristi's fixed point theorem, see [2], [3], [4]. Note that $x_{0}$ is a common fixed point for all admissible mappings.

REMARK 3. In case (2), for any given point $x_{1} \in E$ there is a common fixed point $x_{0}$ such that $x_{0}=f_{n} \circ \cdots \circ f_{1}\left(x_{1}\right)$ for suitable $f_{1}, \ldots, f_{n} \in F$. In fact, it follows from the Proposition that one can obtain $x_{1}<_{F} x_{0}$. In particular, if $F=F^{*}$, then there is $f \in F$ such that $x_{0}=f\left(x_{1}\right)$. This yields Theorem 1.6(a) of [6].

REMARK 4. In case (3), for any given point $x_{1} \in E$ there is a common fixed point $x_{0}$ such that $x_{0}$ is the limit of a sequence $\left(f_{n}^{*}\left(x_{1}\right)\right)_{n \in N}$, where $f_{n}^{*} \in F^{*}$; this follows from the Proposition. If in addition $F=F^{*}$, then there is a sequence $\left(f_{n}\right)_{n \in \mathrm{N}}$ with $f_{n} \in F$ such that $f_{n}\left(x_{1}\right) \rightarrow x_{0}$. This yields Theorem 1.6 (b) of [6].

REMARK 5. The existence of a fixed point $x_{0}$ for a simple continuous admissible mapping $f$ follows easily by a direct argument. In fact, since $f^{n}(x)<_{d, \varphi} f^{m}(x)$ when $n \leqslant m$, and $\varphi$ is decreasing with respect to $<_{d, \varphi}$, we see that the sequence $\left(\varphi\left(f^{n}(x)\right)\right)_{n \in N}$ is a decreasing sequence in $\mathbf{R}$. Since $\varphi$ is bounded below, we have $\varphi\left(f^{n}(x)\right) \rightarrow a$, where $a:=\inf _{n \in \mathrm{N}} \varphi\left(f^{n}(x)\right)$. Therefore, since $d\left(f^{n}(x), f^{m}(x)\right) \leqslant \varphi\left(f^{n}(x)\right)-\varphi\left(f^{m}(x)\right)$ for $n \leqslant m$, it follows that $\left(f^{n}(x)\right)_{n \in N}$ is a Cauchy sequence. By completeness then there is $x_{0} \in E$ such that $f^{n}(x) \rightarrow x_{0}$. By continuity of $f$ we then also have $f^{n}(x) \rightarrow f\left(x_{0}\right)$, i.e., $f\left(x_{0}\right)=x_{0}$. This should be compared with $\S 1.7$ of [6].

The author is grateful to the referee for pointing out some inaccuracies in the original version of this note.

\section{BIBLIOGRAPHY}

1. A. Brøndsted, On a lemma of Bishop and Phelps, Pacific J. Math. 55 (1974), 335-341.

2. __ Fixed points and partial orders, Proc. Amer. Math. Soc. 60 (1976), 365-366.

3. J. Caristi, Fixed point theorems for mappings satisfying inwardness conditions, Trans. Amer. Math. Soc. 215 (1976), 241-251. 
4. J. Caristi and W. A. Kirk, Geometric fixed point theory and inwardness conditions, The Geometry of Metric and Linear Spaces (Conf. Michigan State Univ., 1974), Lecture Notes in Math., vol. 490, Springer-Verlag, New York, 1975, pp. 74-83.

5. R. R. Phelps, Support cones in Banach spaces and their applications, Advances in Math. 13 (1974), 1-19.

6. J. Siegel, A new proof of Caristi's fixed point theorem, Proc. Amer. Math. Soc. 66 (1977), 54-56.

7. J. D. Weston, A characterization of metric completeness, Proc. Amer. Math. Soc. 64 (1977), 186-188.

Institute OF MATHEMatics, UNIVERSITY OF COPENHAGEN, 2100 COPENHAGEN $\varnothing$, DenMaRK 